\title{
SEARCH FOR NEW PHYSICS WITH ATLAS AT THE LHC
}

\author{
V.A. MITSOU \\ CERN, EP Division, CH-1211 Geneva 23, Switzerland \\ and \\ University of Athens, Physics Department, Nuclear and Particle Physics Section, \\ Panepistimioupolis, GR-157 71 Athens, Greece \\ E-mail: Vasiliki.Mitsou@cern.ch \\ On behalf of the ATLAS Collaboration
}

\begin{abstract}
Due to the high energy and luminosity of the LHC, the ATLAS experiment has a huge discovery potential for new physics. A Standard Model Higgs boson can be discovered over the full range of allowed masses, and its mass should be measured with a precision of about $0.1 \%$. The Higgs sector of the MSSM should be fully explored by searches for supersymmetric Higgs bosons. Squarks and gluinos can be discovered up to masses of $2.5 \mathrm{TeV}$ and several precision measurements can be performed in the SUSY sector. The existence of particles predicted by other theories beyond the Standard Model has been also investigated.
\end{abstract}

Presented at the Lake Louise Winter Institute 2000: From Particles to the Universe, Alberta, Canada, 20-26 February 2000.

\section{Introduction}

ATLAS, a general purpose detector for proton-proton collisions, will be capable of exploring the new energy regime of $14 \mathrm{TeV}$ which will become accessible at the Large Hadron Collider (LHC). The LHC will be installed in the existing LEP tunnel at CERN, and will run at a design luminosity of $10^{34} \mathrm{~cm}^{-2} \mathrm{~s}^{-1}$. About $30 \mathrm{fb}^{-1}$ are expected to be collected during the first three years, when the machine will run at low luminosity $\left(10^{33} \mathrm{~cm}^{-2} \mathrm{~s}^{-1}\right)$, while about $100 \mathrm{fb}^{-1}$ per year will be collected when running at design luminosity. An ultimate integrated luminosity of at least $300 \mathrm{fb}^{-1}$ is achievable. The discovery potential of ATLAS for new phenomena is discussed in the following.

\section{Higgs bosons}

The experimental observation of one or several Higgs bosons will be fundamental for a better understanding of the mechanism of electroweak symmetry breaking. In the Standard Model (SM), one doublet of scalar fields is assumed, leading to the existence of one neutral scalar particle H. In the Minimal Su- 
persymmetric Standard Model (MSSM), on the other hand, at least two Higgs doublets are required, corresponding to two charged $\left(\mathrm{H}^{ \pm}\right)$and three neutral (h, H, A) physical states.

\subsection{Standard Model Higgs boson}

The dominant production mechanism of a SM Higgs boson at LHC energies is gluon-gluon fusion, which proceeds via a heavy quark triangle loop. For larger masses, also the WW fusion process contributes significantly.

For Higgs masses below $150 \mathrm{GeV}$, the decay modes to $\mathrm{b} \overline{\mathrm{b}}(B R \simeq 90 \%)$ and to $\tau \tau(B R \simeq 10 \%)$ dominate. The decay to two photons is rather rare $\left(B R \sim 10^{-3}\right)$ and limited to the region $90<m_{\mathrm{H}}<150 \mathrm{GeV}$. At larger masses $(>180 \mathrm{GeV})$, the dominant decays are to $\mathrm{WW}(B R \simeq 75 \%)$ and to $\mathrm{ZZ}(B R \simeq 20 \%)$.

The overall sensitivity for the discovery of a SM Higgs boson is shown in Fig. 1 for various channels assuming an integrated luminosity of $100 \mathrm{fb}^{-1}$. A SM Higgs boson can be discovered with the ATLAS experiment over the full mass range up to $\sim 1 \mathrm{TeV}$ with a high significance. A $5 \sigma$ discovery can be achieved - with two channels in most cases - over the full mass range even after a few years of running at low luminosity.

In the low mass region $\left(m_{\mathrm{H}}<150 \mathrm{GeV}\right)$, a SM Higgs boson can be discovered through the $\mathrm{H} \rightarrow \gamma \gamma$ channel with a signal significance of $5-7 \sigma$ over the continuous $\gamma \gamma$ background. The significance can be further enhanced by exploiting the associated production of the Higgs boson with a $\mathrm{W}$ or a t $\overline{\mathrm{t}}$ pair. In the same mass range, a signal from the $t \bar{t} \mathrm{H}, \mathrm{H} \rightarrow \mathrm{b} \bar{b}$ channel can also be observed with a significance $>5 \sigma$ by exploiting the b-tagging capabilities of the detector.

The decay channel $\mathrm{H} \rightarrow \mathrm{ZZ}^{*} \rightarrow 4 \ell$ provides a rather clean signature in the mass range between $\sim 120 \mathrm{GeV}$ and $2 m_{\mathrm{Z}}$, above which the gold-plated channel with two real $\mathrm{Z}$ bosons in the final states opens up. Both electrons and muons are considered in the final state, thus yielding eeee, ee $\mu \mu$ and $\mu \mu \mu \mu$ event topologies.

In the mass region $150<m_{\mathrm{H}}<180 \mathrm{GeV}$, a pronounced dip occurs in the $B R\left(\mathrm{H} \rightarrow \mathrm{ZZ}^{*}\right)$, due to the opening of the WW decay mode. An excess of events from the $\mathrm{H} \rightarrow \mathrm{WW}^{(*)} \rightarrow \ell \nu \ell \nu$ channel can be used to enhance the significance in this region.

If a SM Higgs boson would be discovered at the LHC using the aforementioned analyses, its mass will be measured with a precision of $0.1 \%$ for $m_{\mathrm{H}}<400 \mathrm{GeV}$ and of $0.1-1 \%$ for $400<m_{\mathrm{H}}<700 \mathrm{GeV}$. The Higgs boson width can be determined for masses above $200 \mathrm{GeV}$ using the $\mathrm{H} \rightarrow \mathrm{ZZ} \rightarrow 4 \ell$ 


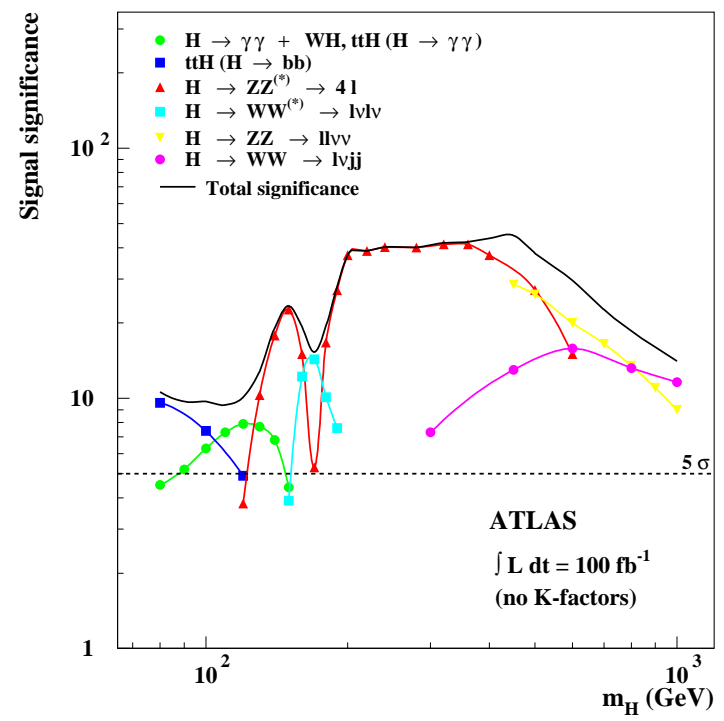

Figure 1. ATLAS sensitivity for the discovery of a Standard Model Higgs boson. The statistical significances are plotted as a function of the Higgs mass for individual channels (different symbols), as well as for the combination of all channels (full line), assuming an integrated luminosity of $100 \mathrm{fb}^{-1}$.

channel.

\subsection{Minimal Supersymmetric Standard Model Higgs}

The capability of the ATLAS experiment to detect MSSM Higgs bosons has been studied in depth over the last few years. Large sparticles masses have been assumed so that Higgs bosons are not allowed to decay to supersymmetric particles. In the MSSM, various decay modes accessible also in the case of the SM Higgs boson are predicted, such as $\mathrm{h} \rightarrow \gamma \gamma, \mathrm{h} \rightarrow \mathrm{b} \overline{\mathrm{b}}, \mathrm{H} \rightarrow \mathrm{ZZ}^{(*)} \rightarrow 4 \ell$. In addition, some channels are strongly enhanced at large $\tan \beta$, e.g. $\mathrm{H} / \mathrm{A} \rightarrow \tau \tau$ and $\mathrm{H} / \mathrm{A} \rightarrow \mu \mu$. Other potentially interesting channels, such as $\mathrm{H} / \mathrm{A} \rightarrow \mathrm{t} \bar{t}$, $\mathrm{A} \rightarrow \mathrm{Zh}, \mathrm{H} \rightarrow$ hh and $\mathrm{H}^{ \pm} \rightarrow$ tb, were also studied. The $5 \sigma$ discovery contours for individual channels in the $\left(m_{\mathrm{A}}, \tan \beta\right)$ plane are shown in Fig. 2 2 for an integrated luminosity of $300 \mathrm{fb}^{-1}$.

Complete coverage of the region shown will be possible at the LHC. Over a considerable fraction of the parameter space, at least two channels are accessible and/or more than one Higgs bosons can be observed. In most cases, 


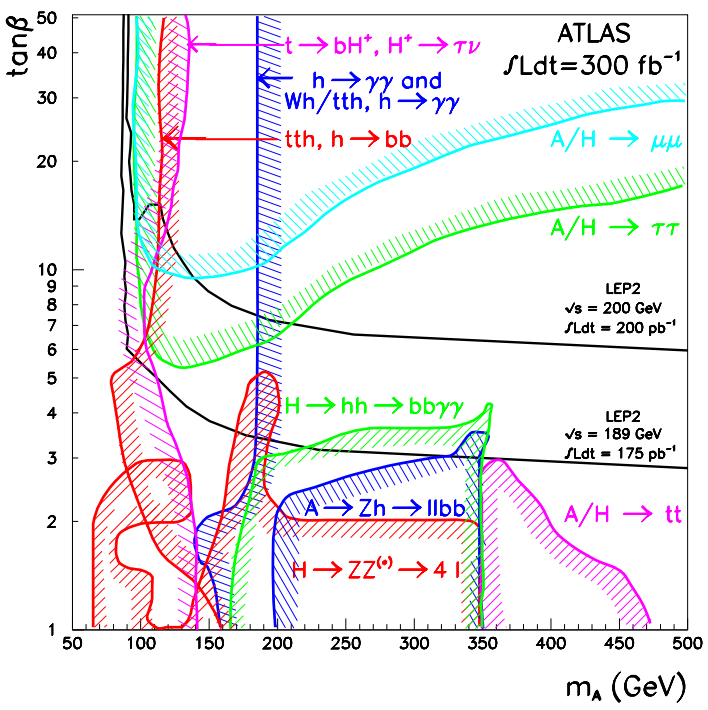

Figure 2. ATLAS sensitivity for the discovery of MSSM Higgs bosons. The $5 \sigma$ discovery contour curves for individual channels are shown in the $\left(m_{\mathrm{A}}, \tan \beta\right)$ plane for an integrated luminosity of $300 \mathrm{fb}^{-1}$.

the experiment will be capable of distinguishing between a SM and an MSSM Higgs boson.

\section{Supersymmetry}

If supersymmetry (SUSY) exists at the electroweak scale, then its discovery at the LHC should be straightforward. The SUSY cross section is dominated by gluinos and squarks production, which are strongly produced with large cross sections. Gluinos and squarks then decay via a series of steps into the LSP (which may itself decay if $R$-parity is violated). These decay chains lead to a variety of signatures involving multiple jets, leptons, photons, heavy flavors (e.g. see Fig. 3), W and Z bosons, and missing energy. The combination of a large production cross section and distinctive signatures makes it easy to separate SUSY from the Standard Model background. Therefore, the main challenge is not to discover SUSY, but to separate the many SUSY processes that occur and to measure the masses and other properties of the SUSY particles. In most cases, the backgrounds from other SUSY events dominate over the reducible SM backgrounds. 


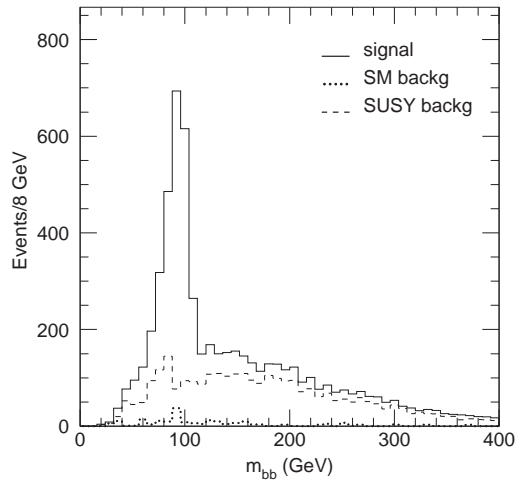

Figure 3. Mass distribution of two b-jets for SUGRA point: $m_{0}=100 \mathrm{GeV}, m_{1 / 2}=$ $300 \mathrm{GeV}, \tan \beta=2.1, A_{0}=300 \mathrm{GeV}$ and $\operatorname{sgn} \mu=+1$. The $\mathrm{h} \rightarrow \mathrm{b} \overline{\mathrm{b}}$ signal (solid), the SUSY background (dashed) and the SM background (dotted) are shown.

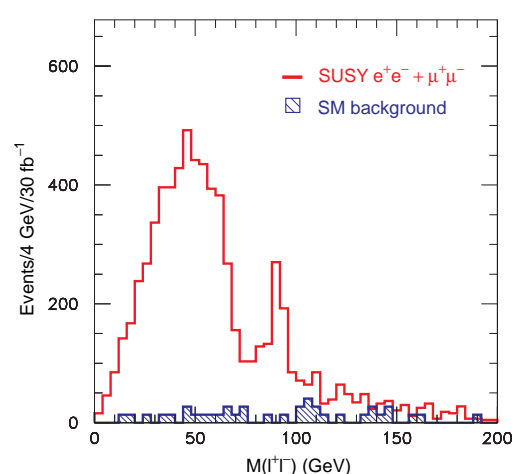

Figure 4. Dilepton distribution for SUGRA point: $m_{0}=800 \mathrm{GeV}$, $m_{1 / 2}=200 \mathrm{GeV}, \tan \beta=10, A_{0}=0$ and $\operatorname{sgn} \mu=+1$. The SUSY signal (solid) and the SM background (shaded) are shown.

The approach followed has been the detailed investigation of signatures for particular points in the parameter spaces of the minimal supergravity (SUGRA), gauge mediated supersymmetry breaking (GMSB) and $R$-parity violating models. Methods such as looking for kinematic endpoints in mass distributions and using these to determine combination of masses have proven generally useful (e.g. see Fig. (1). By using these methods, the fundamental parameters of the underlying theory can be determined with precision of a few percent.

The starting point in the kind of analysis described earlier will be to look for characteristic deviations from the Standard Model. In SUGRA and some other models, there will be events with multiple jets and leptons plus large missing energy. In GMSB models, there would be events with prompt photons or quasi-stable sleptons. In $R$-parity violating models, there would be events with very high jet and/or leptons multiplicities.

\section{Other physics scenarios}

Various theoretical scenarios, in addition to supersymmetry, have been studied by ATLAS in order to establish the discovery potential. ATLAS will be sensitive to new resonances predicted in technicolor theories, up to the $\mathrm{TeV}$ range. Although the parameter space is very large, the number of potential 
channels allows for combinations of signatures to help in understanding the nature of the resonances, and determine the possible existence of techniparticles.

Other studies involve the discovery of excited quarks in the photon plus jet channel (masses up to $6 \mathrm{GeV}$ ), leptoquarks (masses up to $1.5 \mathrm{TeV}$ ) and compositeness probed by the high $p_{\mathrm{T}}$ jets (mass scale up to $40 \mathrm{TeV}$ ). New vector bosons can be discovered through their leptonic decays for masses up to 5-6 TeV. Monopoles can be probed via the $\gamma \gamma \mathrm{X}$ cross section for masses up to $20 \mathrm{TeV}$. It is also possible to investigate extra-dimensions scenarios by searching for missing energy plus jet or missing energy plus photon signatures.

\section{Conclusions}

ATLAS has a wide discovery potential for new physics and sensitivity to a large variety of signatures. A Standard Model Higgs boson can be discovered, if exists, over the whole mass range up to $\sim 1 \mathrm{TeV}$. MSSM Higgs bosons are accessible over a large part of the $\left(m_{\mathrm{A}}, \tan \beta\right)$ parameter space.

Supersymmetric partners are expected to reveal themselves in signatures involving large missing energy, many leptons and/or many jets. These studies have been performed in the context of constrained supersymmetric scenarios, such as supergravity, gauge mediated supersymmetry breaking and $R$-parity violation.

Moreover, the possibility of discovering new physics in many other cases has been investigated by ATLAS, such as technicolor, compositeness and extra dimensions. Limits on the existence of excited quarks, leptoquarks, new gauge bosons, monopoles can be easily set up to the $\mathrm{TeV}$ scale.

\section{Acknowledgments}

I would like to thank the organizers of the Winter Institute for their hospitality and support during my stay at Lake Louise. This work was supported in part by the Greek State Scholarships Foundation (I.K.Y.).

\section{References}

1. ATLAS Collaboration, Detector and Physics Performance Technical Design Report, CERN/LHCC 99-14 (vol. 1) and CERN/LHCC 99-15 (vol. 2), 1999.

http://atlasinfo.cern.ch/Atlas/GROUPS/PHYSICS/TDR/

access.html 